\title{
miR-429 functions as a tumor suppressor by targeting FSCNI in gastric cancer cells
}

\author{
This article was published in the following Dove Press journal: \\ OncoTargets and Therapy \\ 3 March 2016 \\ Number of times this article has been viewed
}

\author{
Min Zhang' \\ Bing-bin Dong ${ }^{2}$ \\ Min Lu' \\ Mei-juan Zheng' \\ He Chen' \\ Jing-zhen Ding ${ }^{3}$ \\ A-Man $X u^{2}$ \\ Yuan-hong $\mathrm{Xu}{ }^{1}$ \\ 'Clinical Laboratory, ${ }^{2}$ Department \\ of General Surgery, The First \\ Affiliated Hospital of Anhui Medical \\ University, Hefei, People's Republic \\ of China; ${ }^{3}$ Department of Cellular \\ and Molecular Medicine, Howard \\ Hughes Medical Institute, University \\ of California at San Diego, La Jolla, \\ CA, USA
}

Correspondence: Yuan-hong Xu Clinical Laboratory, The First Affiliated Hospital of Anhui Medical University, 218 Jixi Road, Hefei, Anhui 230022, People's Republic of China

Tel +86 55I 62922125

Fax +86 55। 62922125

Email xyhong1964@I63.com

A-Man Xu

Department of General Surgery,

The First Affiliated Hospital of Anhui

Medical University, 2 I 8 Jixi Road, Hefei,

Anhui 230022, People's Republic of China

Tel +8655162876038

Fax +8655 I 62876038

Email xuamanI66@sina.com

\begin{abstract}
It has been previously reported that the deregulation of microRNAs in gastric cancer (GC) was correlated with the progression and prognosis. miR-429, a member of the miR-200 family, was previously shown to play an important role in human carcinomas. Our study shows that miR-429 is significantly downregulated in GC tissues compared with matched nontumor tissues. Overexpression of miR-429 in GC cells suppressed cell proliferation. Fascin-1 (FSCN1) was identified as one of the targets of miR-429 and knockdown of FSCN1 mimics the function of miR-429 overexpression. In conclusion, miR-429 acts as a tumor suppressor by targeting FSCN1, suggesting that miR-429 and FSCN1 can both be potential therapeutic targets of GC.
\end{abstract}

Keywords: miR-429, gastric cancer, FSCN1, proliferation

\section{Introduction}

Gastric cancer (GC) is the fourth most common type of cancer causing $\sim 800,000$ mortalities worldwide each year. ${ }^{1}$ In general, patients with GC have a 5-year survival rate of about $15 \%$ and the median overall survival is $<1$ year for the ones with advanced GC. ${ }^{2,3}$ Currently, molecular profiling of GC has suggested that genetic alterations, chromosomal instability, and Helicobacter pylori infections are correlated with GC development and progression. ${ }^{2,4}$ However, the complex molecular mechanisms still need to be fully elucidated. Therefore, it is essential to identify the deregulated genes in GC and elucidate their roles in GC carcinogenesis and progression.

MicroRNAs (miRNAs) are small noncoding RNA of 19-23 nucleotides that negatively regulate gene expression by partial complementary binding, particularly to 3 ' untranslated regions (UTRs) of mRNAs. ${ }^{5}$ MiRNAs have been implicated in the development of acute myeloid leukemia, breast cancer, non-small-cell lung carcinoma, hepatocellular carcinoma, colon cancer, GC, and other cancers. ${ }^{6-10}$ In GC, the deregulated miRNAs and their roles in GC development have also attracted much attention, and a set of miRNAs has been implicated in GC carcinogenesis and progression. ${ }^{11-14}$ Identification of the deregulated miRNAs and their targets may provide promising therapeutic targets for GC treatment.

miR-429, a member of the miR-200 family of miRNAs, was reported able to inhibit invasion in colorectal carcinoma, breast cancer, oral squamous cell carcinoma, and endometrioid adenocarcinoma. ${ }^{15-18}$ However, its upregulation in patients with serous ovarian carcinoma is correlated with survival. ${ }^{19}$ Despite the important role of miR-429 in tumor progress, its precise role and the mechanisms involved have not been addressed so far. In the present study, we have explored the role of miR-429 in GC and identified one of the major targets of this miRNA. We found that miR-429 is significantly downregulated in GC tissues as compared with matched nontumor tissues. We also evaluated the biological influence of overexpression of miR-429 in 
SGC-7901 cells. Furthermore, we identified and validated fascin-1 (FSCN1) as one of the targets of miR-429, which suggests that miR-429 participates in cancer cell biology by regulating FSCN1 expression.

\section{Methods}

\section{Cell lines and patient samples}

SGC-7901 (a human gastric carcinoma cell line) was purchased from the Type Culture Collection of the Chinese Academy of Sciences (Shanghai, People's Republic of China). Cells were maintained in RPMI-1640 medium (Hyclone, Logan, Utah, USA) containing $10 \%$ fetal bovine serum and $1 \%$ penicillin/streptomycin at $37^{\circ} \mathrm{C}$ in a $5 \% \mathrm{CO}_{2}$ incubator.

Tissue samples and paired adjacent nontumor tissue samples were collected from the surgical specimens of 44 patients with gastric carcinoma from the First Affiliated Hospital of Anhui Medical University. This study was approved by the ethics committee of the First Affiliated Hospital of Anhui Medical University and informed consent was obtained from each patient.

\section{RNA extraction and quantitative real-time PCR}

Total RNA was extracted from cultured cells or tissue samples using TRIzol Reagent (Thermo Fisher Scientific, Waltham, MA, USA) as per the manufacturer's instruction. The miR-429 level was quantified by quantitative reverse transcription-PCR (qRT-PCR) using Taqman assay kit (Thermo Fisher Scientific, 4366596) and Realtime PCR Master Mix (QPK-101, Toyobo, Shanghai, People's Republic of China), with U6 small nuclear RNA as internal normalized reference. For FSCN1 mRNA quantification, cDNA was synthesized by using ReverTra Ace qPCR RT Master Mix with gDNA Remover (Toyobo, FSQ-301). And it was quantificated by KAPA SYBR FAST Universal qPCR kit (Kapa Biosystems, KK4601, Wilmington, Massachusetts, USA) using $18 \mathrm{~s}$ as internal reference. The data were measured using an ABI 7900 HT PCR sequencer (Applied Biosystems), and gene expression was calculated using the $\Delta \Delta \mathrm{Ct}$ method. All reactions were performed in triplicate.

\section{Plasmid construction}

For the luciferase reporter assay, a wild-type 3'UTR of FSCN1 mRNA (Genbank accession no NM_003088.3) was amplified and cloned into the XhoI/NotI site just after the Renilla luciferase reporter gene of the luciferase reporter vector psiCHECK-2, which was named FSCN1-3'UTRpsiCHECK2-WT. FSCN1-3'UTR-psiCHECK2-MUT carried the mutated sequence in the complementary site for the seed region of miR-429 and was generated based on FSCN13'UTR-psiCHECK 2-WT by site-specific mutagenesis (Transgene, Beijing, People's Republic of China), which mutated CAGTATT to AGTGCGA.

For FSCN1-overexpressing plasmid, the human FSCN1CDS was amplified using the primers as follows: $5^{\prime}$-TAAG AATTCCGCGCAGCGGCCTCTCGTCTAC-3' (forward) and 5'-TAAGGATCCGTTAGCAGGGAGGGTTGGCAG GAGC-3' (reverse) and cloned into EcoRI/BamHI site of pcDNA3.1-vector.

\section{Transient transfection with miRNA/ siRNA/plasmid}

miR-429 mimics was purchased from Shanghai GenePharma Co., Ltd. (Shanghai, People's Republic of China), and siRNA targeting FSCN1 mRNA (NM_003088.3) was purchased from Sigma-Aldrich Co. (St Louis, MO, USA). Transfection of miRNA mimics or plasmid was conducted using $3 \mu \mathrm{L}$ Lipofectamine 2000 (Thermo Fisher Scientific). Transfection of siRNA was performed using $3 \mu \mathrm{L}$ SuperFectin II DNA Transfection Reagent (Pufei, Shanghai, People's Republic of China). In the rescue experiment, cells were cotransfected with $50 \mathrm{nM}$ miRNA mimics and $2 \mu \mathrm{g}$ overexpression plasmid using $4 \mu \mathrm{L}$ Lipofectamine 2000.

\section{EdU assay}

A total of $1.2 \times 10^{5}$ transfected cells were plated into 6-well plates, and DNA synthesis was assessed with a Cell-Light ${ }^{\mathrm{TM}}$ EdU Apollo ${ }^{\circledR} 488$ In Vitro Imaging Kit (C10310-3, RiboBio, Guangzhou, People's Republic of China). Then, nuclei were stained with DAPI (Thermo Fisher Scientific). Finally, cells were visualized and counted with a fluorescent microscope (Leica Microsystems, Wetzlar, Germany). The EdU labeling index was calculated as EdU-positive cells/total DAPI-positive cells.

\section{Dual luciferase reporter assay}

For luciferase reporter assay, SGC-7901 cells were cotransfected with miR-429 mimics and plasmid. The luciferase activity was measured using the Dual Luciferase Reporter Assay System (Promega Corporation, Fitchburg, WI, USA). The relative LUC means the ratio of Fluc(the value of firefly fluorescence) and Rluc(the value of renilla fluorescence). Transfections were done in duplicate and repeated at least thrice in independent experiments.

\section{Western blot analysis}

Cells were harvested 48 hours post-transfection and lysed in RIPA buffer (Beyotime, Shanghai, People's Republic of 
China). Total proteins from tissue samples were extracted by grinding with liquid nitrogen. Proteins were separated by a $10 \%$ polyacrylamide gel and transferred to a PVDF membrane, then detected with anti-Fascin antibody (EMD Millipore, Billerica, MA, USA, MAB3582). $\beta$-Actin was used as the control. The goat antimouse immunoglobulin $\mathrm{G}$ (Licor Co., Lincoln, NE, USA) was used as the secondary antibody. The membranes were scanned with an Odyssey LI-CDR scanner (BD Biosciences, San Jose, CA, USA).

\section{Immunohistochemistry}

Sections were dewaxed in xylene, rehydrated in alcohol, and immersed in 3\% hydrogen peroxide for 5 minutes to suppress endogenous peroxidase activity. Antigen retrieval was performed by heating at $100^{\circ} \mathrm{C}$ for 30 minutes in $0.01 \mathrm{~mol} / \mathrm{L}$ sodium citrate buffer ( $\mathrm{pH}$ 6.0). After three rinses, sections were incubated for 1 hour in room temperature with antiFascin antibody (Millipore). Then sections were incubated with biotin-labeled secondary antibody. After three additional washes, slides were visualized with Horseradish Peroxidase Color Development Kit (DAB) and counterstained with hematoxylin, then observed under the microscope.

\section{MTT assay}

SGC-7901 cells were seeded in 96-well plates (1,000 cells/ $100 \mu \mathrm{L}$ medium/well) after transfection. Cells were incubated for $1,2,3,4$, and 5 days and then incubated for 4 hours in the presence of $20 \mu \mathrm{L}$ MTT solution ( $5 \mathrm{~g} / \mathrm{L}$, Sigma-Aldrich Co.). An amount of $150 \mu \mathrm{L} /$ well DMSO was added and OD values at $490 \mathrm{~nm}$ were recorded. The assay was performed three times.

\section{Colony formation assay}

SGC-7901 cells were treated with oligo RNA and the cells were harvested after 24 hours. The cells were suspended and plated in 6-well plates ( 800 cells $/ 2 \mathrm{~mL}$ medium/well). After being cultured for 7 days, colonies were stained with $0.1 \%$ crystal violet and pictured.

\section{Xenograft tumor model}

Approximately $10^{6}$ SGC-7901 cells infected with miR-429-LV were subcutaneously injected into left flanks of nude mice, whereas the SGC-7901/NC-LV cells were inoculated into the right side. Five mice were included in each group. The volume of the implanted tumor was measured every 4 days, using the formula: volume $\left(\mathrm{mm}^{3}\right)=0.5 \times$ length $\times$ width ${ }^{2}$. The mice were killed and the tumors were weighed 25 days after injection.
The 4-5-week-old mice were purchased from Shanghai Super-B\&K Laboratory Animal Corp. Ltd. (Shanghai, People's Republic of China). The animal study was carried out in strict accordance with the recommendations in the guidelines for the Animal Care and Use Committee of the First Affiliated Hospital of Anhui Medical University.

\section{TCGA dataming}

The public TCGA (http://cancergenome.nih.gov/) data were used as our primary source of samples. Gene-level summarized (level 3, RPKM and read count) RNA-seq data sets were used. In total, 249 tumors and 33 normal tissues having expression data (date of download: November 2014) were profiled for different expression analysis. DESeq, an $\mathrm{R}$ Bioconductor package, was used to test for differentially expressed genes from patient data. TargetScan version 6.2 human miRNA target predictions were obtained online (http://targetscan.org).

\section{Statistical analysis}

All statistical analyses were performed by using the SPSS 18.0 version (SPSS Inc., Chicago, IL, USA). Data were tested using two-tailed Student's $t$-test and one-way ANOVA. $P<0.05$ was considered to be statistically significant.

\section{Results miR-429 is downregulated in GC tissues}

To explore the expression of miR-429 in GC, we analyzed 44 paired GC tumors and non-neoplastic tissues from GC patients (the features of patients are shown in Table S1) by performing quantitative real-time RT-PCR (qRT-PCR). As shown in Figure 1A and B, the expression of miR-429 was downregulated in $63.6 \%$ (28 of 44 ) of GC tissues as compared with that in their matched controls.

\section{Overexpression of miR-429 reduces GC growth both in vitro and in vivo}

Given that the expression of miR-429 is downregulated in $\mathrm{GC}$, we then determined whether miR-429 functions as a tumor suppressor. The cell proliferation was examined by MTT and colony formation assays. We found that the proliferation of cells expressing miR-429 mimics was significantly decreased as compared with that of negative control (NC) miRNA (Figure 1C). The capacity of colony formation was also evaluated on SGC-7901 cells expressing miR-429 mimics, and the results showed that miR-429 significantly reduced the cells' proliferation (Figure 1D). EdU staining results also showed that the proportion of cells at $\mathrm{S}$ and G2 
A

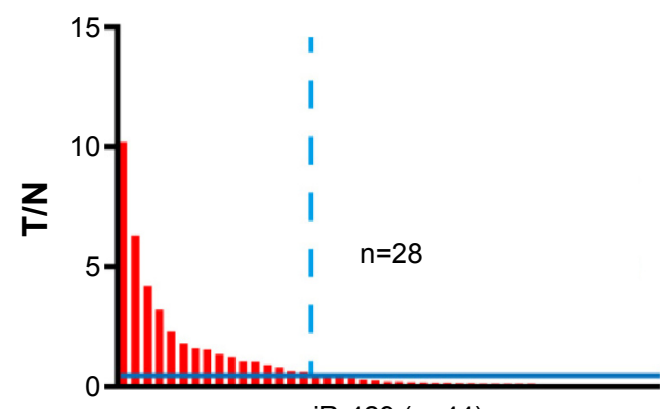

$\operatorname{miR}-429(n=44)$
B

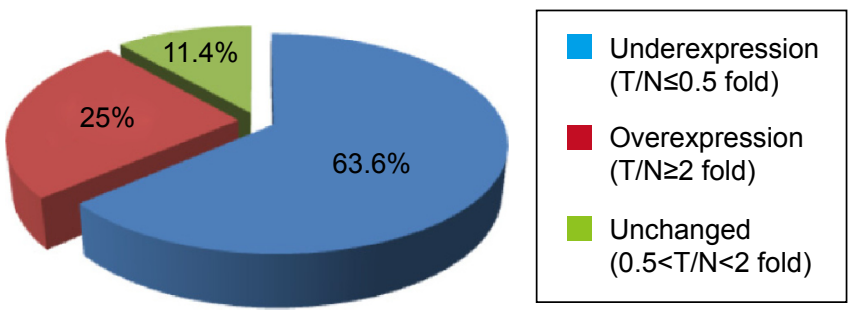

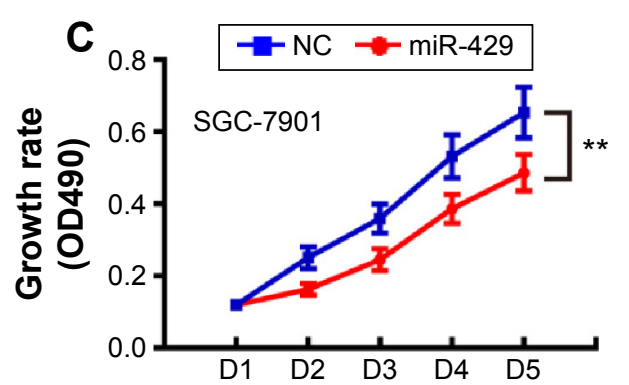

D

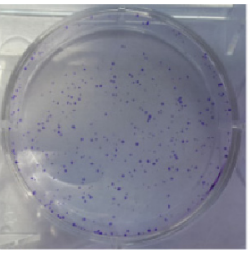

NC

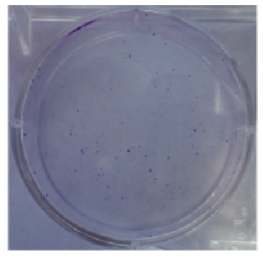

miR-429

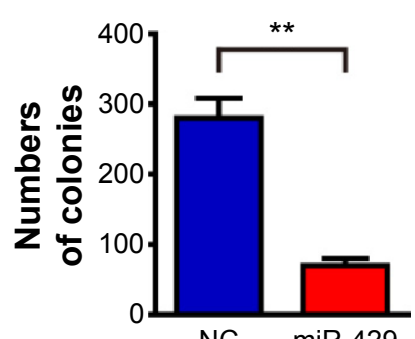

NC miR-429
E

DAPI
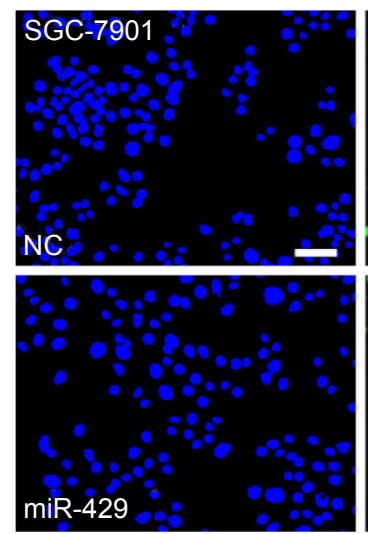

EdU
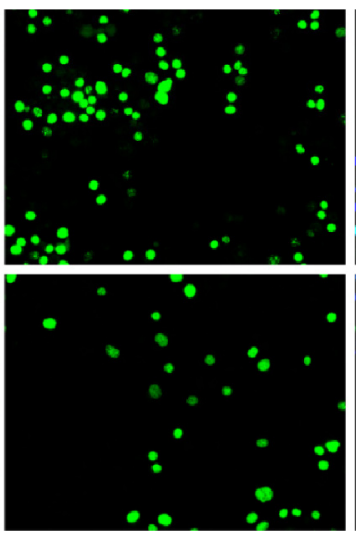

EdU/DAPI

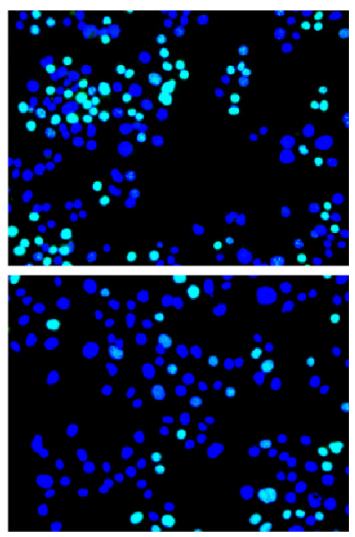

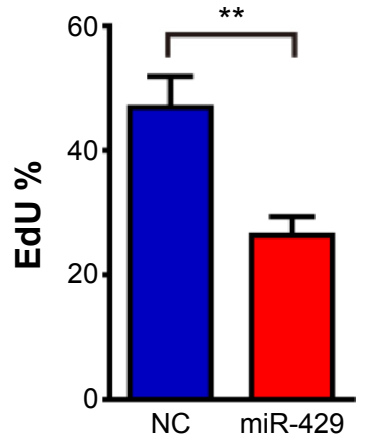

Figure I miR-429 is downregulated in GC and reduces GC cancer cell growth rate in vitro.

Notes: (A) The fold changes of the relative expression of miR-429 (T/N) were assessed by qRT-PCR in 44 pairs of human gastric cancer (GC) specimens (T) and their corresponding normal tissues $(\mathrm{N})$. miR-429 expression of each tissue was calculated by $\Delta \Delta \mathrm{Ct}$ method for relative quantization using $U 6$ as endogenous reference genes. In each pair of clinical sample, the miR-429 expression of normal tissue was used as reference of its corresponding tumor tissue and set as I. The samples were divided into two groups at $\mathrm{T} / \mathrm{N}=0.5$ (blue solid line): 28 pairs with $\mathrm{T} / \mathrm{N}$ ratio below 0.5 -fold were presented on the right side of the blue dashed line, and the others 16 with $\mathrm{T} / \mathrm{N}$ ratio above 0.5 -fold were grouped on the left side of the blue dashed line. (B) The distribution of miR-429 expression in clinical specimens was divided into three parts by $\mathrm{T} / \mathrm{N}=0.5$ and T/N =2 in the pie diagram. (C) Growth curves were plotted at various time points by MTT assay in SGC-790I cells overexpressing miR-429 mimics (miR-429) or negative control (NC) microRNA. The OD values represented the growth rate. DI, D2, D3, D4, and D5 are mean day I, day 2, day 3, day 4, and day 5. (D) Representative dishes of crystal violet-stained colonies were photographed (left) and the colony quantification (right) was presented as colony number per dish. $* * P<0.01$. (E) The nuclei of cells were stained with DAPI (blue) and the cells during S-phase were assessed with EdU (green). EdU \% which means EdU labeling index (\%) was calculated as EdU positive cells/total DAPI-positive cells. ${ }^{* * P}<0.01$.

phase is less in the cells expressing miR-429 than in those with NC, which indicates that miR-429 reduces cell dividing (Figure 1E). To further confirm the biological function of miR-429, an in vivo model was used. Nude mice injected with SGC-7901/miR-429-LV cells showed a significant reduction in tumor size and weight as compared with those of NC $(P<0.01)$ (Figure $2 \mathrm{~A}-\mathrm{C})$.

\section{miR-429 directly targets FSCNI expression}

A high-confidence list of 656 candidate miR-429 targets was obtained by choosing evolutionary conservation (TargetScan probability of conserved targeting $>0.5$ ). It is recognized that target prediction tools quite often yield many false positive targets. Therefore, predicted targets associated with 


\section{A SGC-7901}

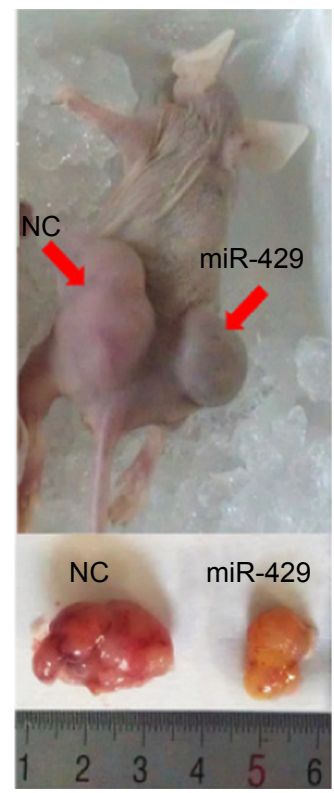

Figure 2 miR-429 reduces gastric cancer (GC) cell growth rate in vivo. Notes: (A) Stable SGC-790I/NC-LV (negative control [NC]) and SGC-790I/miR429-LV (miR-429) cells were inoculated into left and right flanks of each nude mouse (upper). The mice were euthanized and the tumors were excised (bottom). (B and C) The volume $\left(\mathrm{mm}^{3}\right)$ and weight $(\mathrm{mg})$ of each group were measured. The average tumor volume and weight were indicated as mean \pm standard error. Results are representative of three independent experiments.

tumor progression were analyzed (Figure S2) and further validated by examining their expression at the mRNA level upon the transfection of miR-429 in SGC7901 cells. Among these six candidates, the level of FSCN1 was suppressed by
miR-429 (Figure 3A) and the potential 3'UTR binding site of FSCN1 of miR-429 is shown in Figure 3B. To further verify whether miR-429 targets FSCN1 directly, the WT 3'UTR or the MUT 3'UTR (the putative miR-429 binding site was mutated) of FSCN1 was cloned into the reporter vector and the dual luciferase reporter assay was performed. As shown in Figure 3C, the miR-429 mimics the reduced renilla luciferase activity of the wild-type plasmid (wt FSCN1 $3^{\prime} \mathrm{UTR}$ ) which resulted in the increase of relative LUC (Fluc/ Rluc)\% $(P<0.05)$ but not the mutant plasmid (mut FSCN1 $\left.3^{\prime} \mathrm{UTR}\right)$. Moreover, the expression level of FSCN1 protein in the cells overexpressing miR-429 mimics was dramatically lower than that of NC miRNA (Figure 3D). These findings suggest that miR-429 directly targets FSCN1 expression by binding to the 3'UTR region of FSCN1.

\section{Knockdown of FSCNI mimics the effects of miR-429 overexpression}

To further study the role of FSCN1 in GC development, we investigated whether RNA interference knockdown of FSCN1 could recapitulate the oncogenic effects of miR429 in SGC-7901 cells. As shown in Figure 4A-C, FSCN1 knockdown, similar to the transfection of miR-429, significantly reduced cell proliferation and slowed cell cycle progression in SGC-7901 cells. We then performed rescue experiments to further validate that FSCN1 targeting is involved in miR-429-mediated tumor suppression in GC.

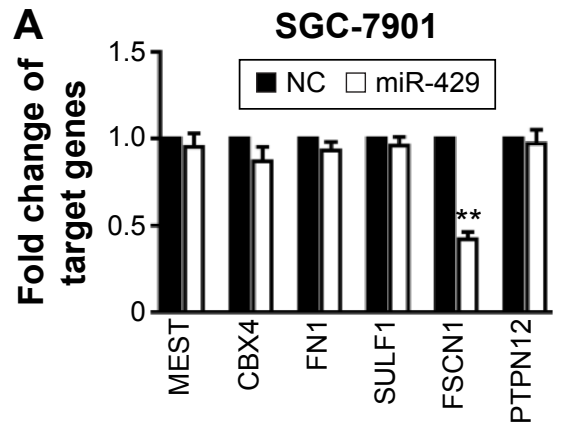

C

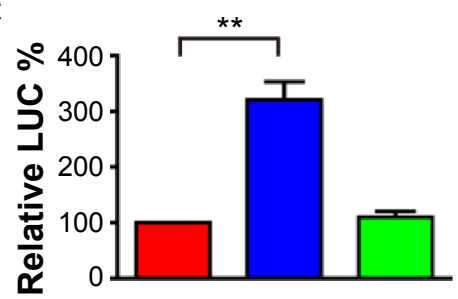

B

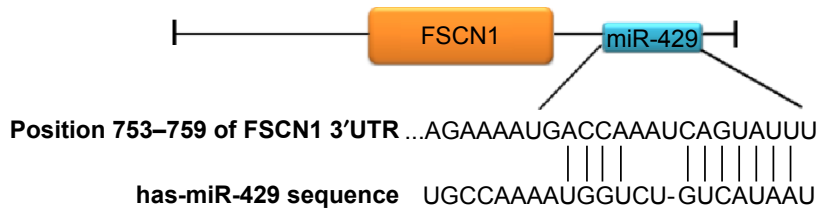

D

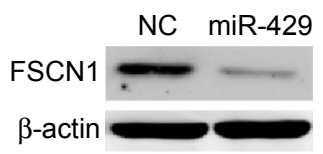

Figure 3 miR-429 directly targets fascin-I (FSCNI).

Notes: (A) Six predicted target genes were verified by examining the expression upon the transfection of miR-429 in SGC-790I cells. $18 \mathrm{~s} r R N A$ was used as internal control (negative control [NC] as 100\%). $* * P<0.01$. (B) The binding site between miR-429 and FSCN/ 3' untranslated region (UTR) was identified by TargetScan. (C) SGC-790I cells were cotransfected with wt or mut FSCNI 3'UTR reporter plasmid and miR-429 or NC. The luciferase activity was measured by dual luciferase reporter assay and presented as relative LUC (firefly/renilla luciferase). The relative LUC of control was set as $100 \%$. Each experiment was repeated at least three times. $* * P<0.01$. (D) The protein level of FSCNI was measured by Western blot. $\beta$-Actin serves as an internal control. 
A

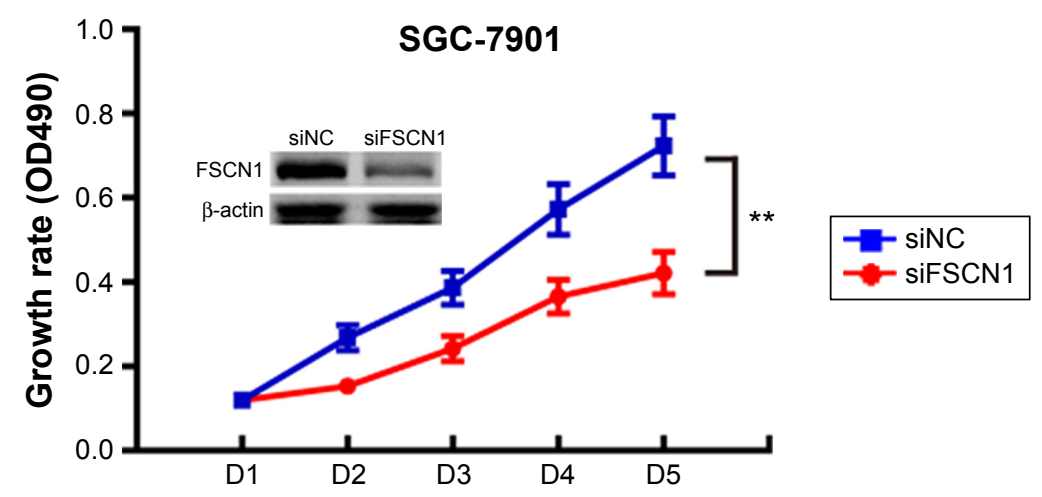

B
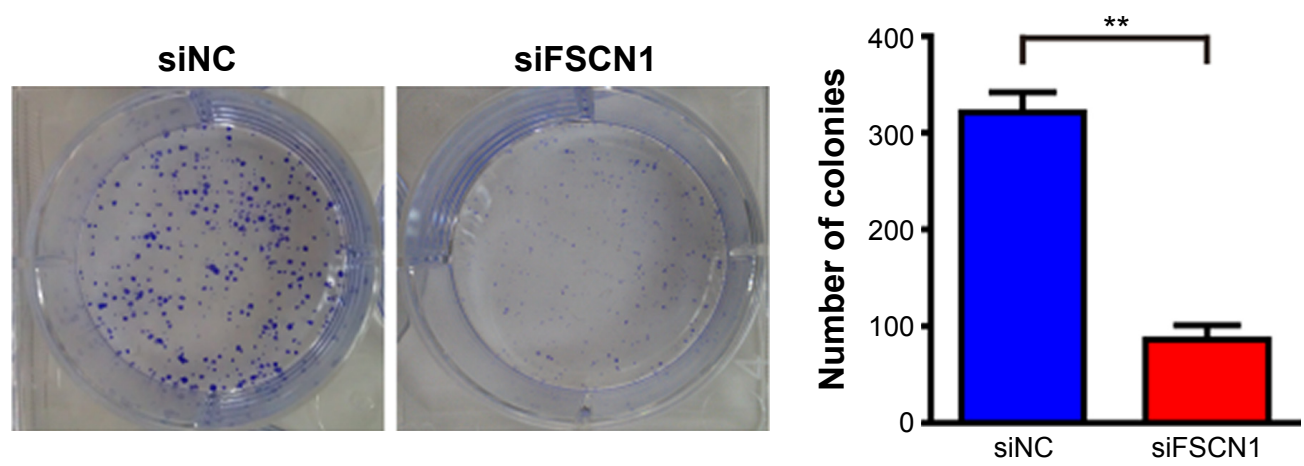

C DAPI
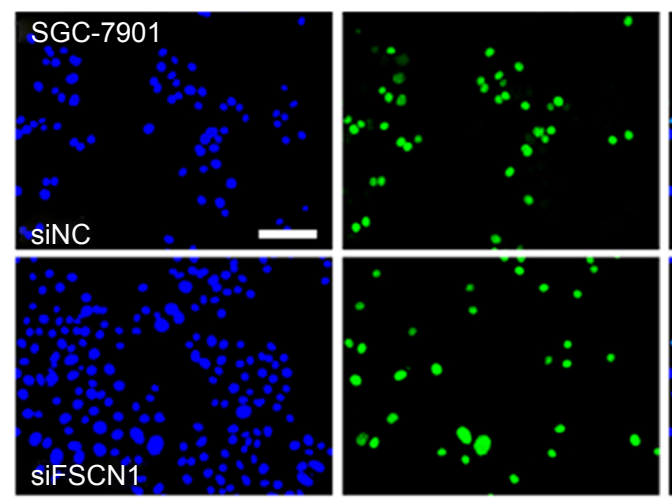

\section{EdU/DAPI}

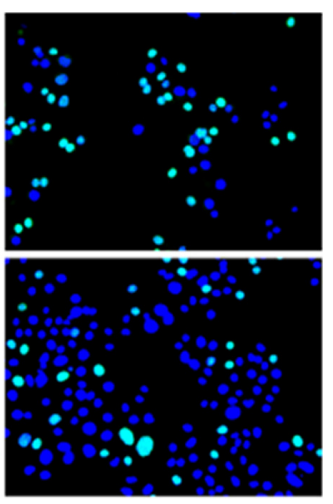

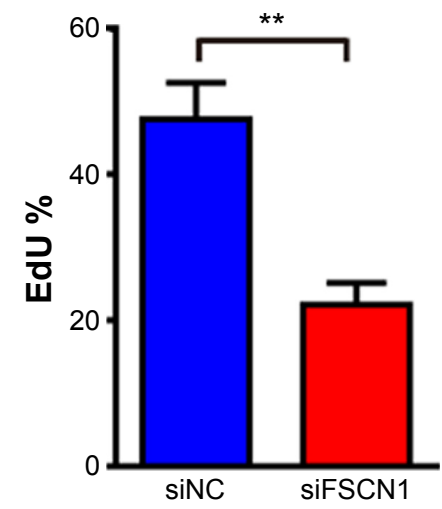
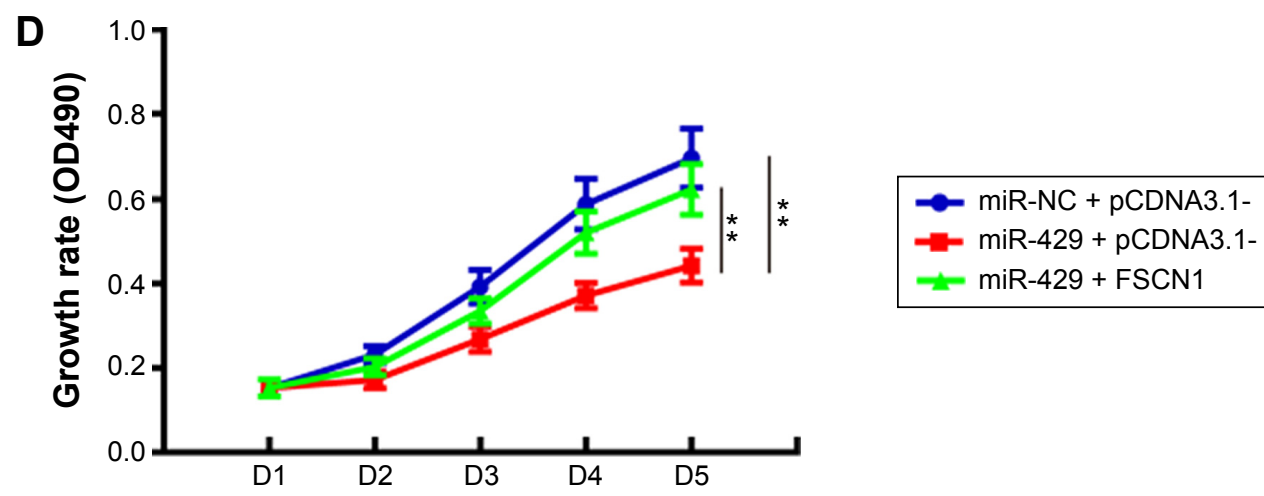

Figure 4 Fascin-I (FSCNI) depletion mimics the effect of miR-429 and its overexpression rescues the growth defect of miR-429.

Notes: (A-C) SGC-790I cells were transfected with siFSCNI or negative control (siNC) and then processed with (A) MTT assay at different time points, DI, D2, D3, D4, and D5 (mean day I, day 2, day 3, day 4, and day 5) (B) colony formation assay. Left, representative plates. Right, quantitation of colony numbers. $* * P<0.0$ I. (C) EdU assay. The EdU labeling index is shown (right). Results are representative of three independent experiments. $* * P<0.0 \mathrm{I}$. The efficiency of siFSCNI is shown in (A) (top left). Student's $t$-test was used to obtain the P-value. (D) FSCNI expression rescues the growth defect in cells transfected with miR-429. The growth rate was promoted by the overexpression of FSCNI in miR-429-overexpressing cells. The data were obtained using the MTT assay at different timepoints after transfection. **P $<0.0 \mathrm{I}$. 
A

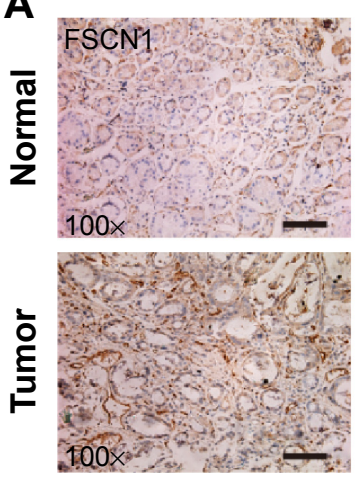

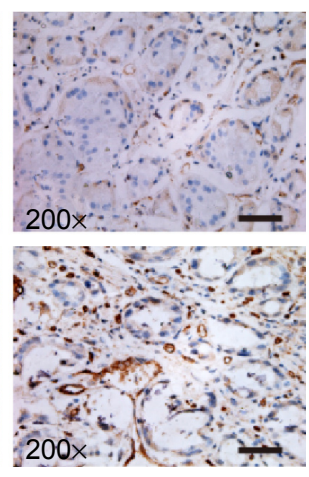

B

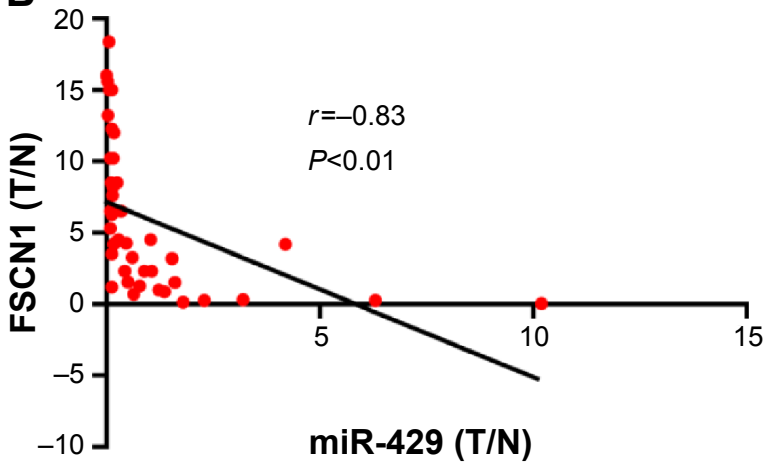

Figure 5 The correlation between fascin-I (FSCNI) and miR-429 in clinical specimens.

Notes: (A) Representative IHC photos of FSCNI expression in normal and tumor tissues. The scale bars of $100 \times$ and $200 \times$ respectively represented 200 and $100 \mu$ m. (B) The relative expression of miR-429 (T/N) was inversely correlated with FSCNI (T/N) expression $(r=-0.83, P<0.01)$. Expression levels in 44 pairs of human gastric carcinoma specimens $(\mathrm{T})$ and their corresponding normal tissues $(\mathrm{N})$ were quantified using qRT-PCR. miR-429 was normalized to U6 and FSCNI was normalized to I8 s and analyzed with the Pearson correlation. Each red dot represents a pair of specimens. For comparison, a two-tailed, unpaired Student's $t$-test was used. Correlation coefficient $r=-0.83 ; P<0.01$.

FSCN1 overexpression vector was used in the experiments. As shown in Figure 4D, overexpression of FSCN1 protein significantly restores the growth defect in SGC-7901 cells transfected with miR-429.

To confirm the clinic relevance of our in vitro findings, we also used immunohistochemical analysis to evaluate the FSCN1 level in GC and an increased expression of FSCN1 in GC was observed (Figure 5A). Besides, FSCN1 mRNA expression was also evaluated in GC samples. As shown in Table 1, FSCN1 mRNA expression was upregulated in 68.06\% (28 of 44) of GC samples, and an inverse correlation with miR-429 expression in GC samples was noticed (Figure 5B, $r=-0.83$; $P<0.01)$. These findings further suggest that decreased expression of miR-429 leads to the upregulation of FSCN1 in GC and indicate that a reduction of FSCN1 expression can mimic miR-429 in promoting SGC-7901 cells to proliferate.

\section{Discussion}

The role of miRNA in tumorigenesis has been extensively studied in recent years. ${ }^{7,14}$ miR-429, a member of the miR-200

Table I The relative expression of miR-429 was quantified using qRT-PCR and normalized by $\mathrm{U} 6$ and fascin-I (FSCNI) was evaluated by immunohistochemical $(\mathrm{IHC})$ staining. IHC scores of FSCNI in miR-429 low or miR-429 high group

\begin{tabular}{llll}
\hline & FSCN I (-) & FSCN I (+) & Total \\
\hline miR-429 low & 8 & 25 & 33 \\
miR-429 high & 8 & 3 & $1 \mathrm{I}$ \\
Total & 16 & 28 & 44 \\
\hline
\end{tabular}

Notes: The histologic appearance and staining intensity were examined and scored by two pathologists independently and classified as follows: absent (scored as -), weak-positive (scored as + ), moderately-positive (scored as ++ ), and strong-positive (scored as +++). We classified ++ or +++ expression as positive (FSCNI [+]), and + or - expression as negative (FSCNI $[-]) . P=0.011$. family of miRNAs, was downregulated in various types of cancer, including oral squamous cell carcinoma, ${ }^{17}$ esophageal carcinomas, ${ }^{18}$ hepatitis $\mathrm{B}$ virus-related hepatocellular carcinoma, ${ }^{20}$ breast cancer, ${ }^{16}$ colorectal cancer, ${ }^{21}$ and GC. ${ }^{22} \mathrm{~A}$ dynamic negative correlation between miR-429 expression and the colorectal cancer progression was observed as well. ${ }^{21}$ However, miR-429 was also reported to be upregulated in human colorectal cancer tissues and the miR-429 overexpression suppressed cell apoptosis in HT-29 cells. ${ }^{23}$ The downstream targets identified are also complex, which seemed to be tissue-dependent. For example, miR-429 inhibits expression of the transcriptional repressors ZEB1 in breast cancer and oral squamous cell carcinoma. ${ }^{16,17}$ Downregulation of miR-429 in colorectal carcinoma contributes to carcinogenesis by targeting Onecut2. ${ }^{15}$ Upregulation of miR-429 inhibits invasion and promotes apoptosis in esophageal carcinoma cells by targeting Bcl-2 and SP1 in esophageal carcinoma. ${ }^{18}$ miR-429 also modulates the expression of c-myc in human gastric carcinoma cells. ${ }^{22}$ Therefore, additional downstream targets need to be discerned for a more comprehensive understanding of the role of miR-429 in cancer progression, by which it would guide the design of future personalized medicine for cancer patients.

In this study, we showed that miR-429 was significantly downregulated in GC tumor tissues when compared with adjacent normal tissue, which was consistent with a previous study. ${ }^{22}$ We further used in vitro proliferation assay and in vivo mice model, by which we confirmed the tumor suppressing role of miR-429 in GC cells. To elucidate the underlying molecular mechanisms of miR-429 in cancer cell proliferation, the public TCGA data, gene-level summarized RNA-seq data sets, DESeq, and TargetScan were used. By R package, 
a total of 168 genes were selected as the different expression genes between tumor and normal samples, of which 127 were upregulated. As shown in Figure S1, FSCN1 emerged as one of the most prominent upregulated genes in the TCGA stomach adenocarcinoma dataset by hierarchical clustering analysis. Then we demonstrated that the knockdown of FSCN1 mimics the effects of miR-429 overexpression that leads to the growth defect in GC cells. Furthermore, FSCN1 overexpression reversed the inhibitory effect of miR-429 on GC proliferation. In order to seek the clinical relevance, we also evaluate the expression level of FSCN1 in GC tissues and found that its expression is upregulated in GC and negatively correlated with the level of miR-429.

FSCN1, a $55 \mathrm{kDa}$ actin-binding protein, is an important regulatory element in the maintenance and stability of parallel bundles of filamentous actin and plays a central role in the regulation of cell adhering, migration, and invasion. ${ }^{24,25}$ The overexpression of FSCN1 was associated with aggressive clinical course, poor prognosis, and shorter survival of various tumors including prostate, breast cancer, gastric, esophageal, and pancreatic cancer, ${ }^{26-31}$ indicating that FSCN1 may play an important role in tumorigenesis. The tumorigenic function of FSCN1 might be conferred by its invasive properties on cancer cells. ${ }^{32}$ In particular, the knockdown of FSCN1 expression reduced the proliferation and metastasis of $\mathrm{GC}$ cells $\mathrm{s}^{33}$ and the higher expression of FSCN1 is correlated with more advanced cancer stages and inversely with survival rates in gastric adenocarcinomas. ${ }^{34}$ A previous study identified FSCN1 as a target of miR-133b in GC cells. ${ }^{35}$ In our study, we indicated that FSCN1 was also a direct target of miR-429 in GC cells and demonstrated the inverse correlation of FSCN1 and miR-429 in vivo. So, we speculate that miR-429 may contribute to the inhibition of GC via directly targeting FSCN1 and that miR-429 may serve as a useful biomarker for earlier diagnosis and prognosis of GC.

\section{Acknowledgment}

The study was supported by the Natural Science Foundation of Anhui Province, People's Republic of China (No 1301043022).

\section{Disclosure}

The authors report no conflicts of interest in this work.

\section{References}

1. Ferro A, Peleteiro B, Malvezzi M, et al. Worldwide trends in gastric cancer mortality (1980-2011), with predictions to 2015, and incidence by subtype. Eur J Cancer. 2014;50(7):1330-1344.
2. Bang YJ. Advances in the management of HER2-positive advanced gastric and gastroesophageal junction cancer. J Clin Gastroenterol. 2012; 46(8):637-648.

3. Delaunoit T. Latest developments and emerging treatment options in the management of stomach cancer. Cancer Manag Res. 2011;3: 257-266.

4. Kim KM, Kwon MS, Hong SJ, et al. Genetic classification of intestinaltype and diffuse-type gastric cancers based on chromosomal loss and microsatellite instability. Virchows Archiv. 2003;443(4):491-500.

5. Fabian MR, Sonenberg N, Filipowicz W. Regulation of mRNA translation and stability by microRNAs. Annu Rev Biochem. 2010;79: 351-379.

6. Zheng YS, Zhang H, Zhang XJ, et al. MiR-100 regulates cell differentiation and survival by targeting RBSP3, a phosphatase-like tumor suppressor in acute myeloid leukemia. Oncogene. 2012;31(1):80-92.

7. Nicoloso MS, Spizzo R, Shimizu M, Rossi S, Calin GA. MicroRNAs the micro steering wheel of tumour metastases. Nat Rev Cancer. 2009; 9(4):293-302.

8. Mattiske S, Suetani RJ, Neilsen PM, Callen DF. The oncogenic role of miR-155 in breast cancer. Cancer Epidemiol Biomarkers Prev. 2012; 21(8):1236-1243.

9. Malleter M, Jacquot C, Rousseau B, et al. miRNAs, a potential target in the treatment of non-small-cell lung carcinomas. Gene. 2012; 506(2):355-359.

10. Hou YY, Cao WW, Li L, et al. MicroRNA-519d targets MKi67 and suppresses cell growth in the hepatocellular carcinoma cell line QGY7703. Cancer Lett. 2011;307(2):182-190.

11. Chan SH, Wu CW, Li AF, Chi CW, Lin WC. miR-21 microRNA expression in human gastric carcinomas and its clinical association. Anticancer Res. 2008;28(2A):907-911.

12. Peng W, Chen ZY, Wang L, Wang Z, Li J. MicroRNA-199a-3p is downregulated in gastric carcinomas and modulates cell proliferation. Genet Mol Res. 2013;12(3):3038-3047.

13. Tsukamoto Y, Nakada C, Noguchi T, et al. MicroRNA-375 is downregulated in gastric carcinomas and regulates cell survival by targeting PDK1 and 14-3-3zeta. Cancer Res. 2010;70(6):2339-2349.

14. $\mathrm{Wu} \mathrm{WK}$, Lee $\mathrm{CW}$, Cho $\mathrm{CH}$, et al. MicroRNA dysregulation in gastric cancer: a new player enters the game. Oncogene. 2010;29(43): 5761-5771.

15. Sun Y, Shen S, Liu X, et al. MiR-429 inhibits cells growth and invasion and regulates EMT-related marker genes by targeting Onecut 2 in colorectal carcinoma. Mol Cell Biochem. 2014;390(1-2):19-30.

16. Ye ZB, Ma G, Zhao YH, et al. miR-429 inhibits migration and invasion of breast cancer cells in vitro. Int J Oncol. 2015;46(2):531-538.

17. Lei W, Liu YE, Zheng Y, Qu L. MiR-429 inhibits oral squamous cell carcinoma growth by targeting ZEB1. Med Sci Monit. 2015;21: 383-389.

18. Wang Y, Li M, Zang W, et al. MiR-429 up-regulation induces apoptosis and suppresses invasion by targeting Bcl-2 and SP-1 in esophageal carcinoma. Cell Oncol. 2013;36(5):385-394.

19. Nam EJ, Yoon H, Kim SW, et al. MicroRNA expression profiles in serous ovarian carcinoma. Clin Cancer Res. 2008;14(9):2690-2695.

20. Gao H, Liu C. miR-429 represses cell proliferation and induces apoptosis in HBV-related HCC. Biomed Pharmacother. 2014;68(8):943-949.

21. Sun Y, Shen S, Tang H, et al. miR-429 identified by dynamic transcriptome analysis is a new candidate biomarker for colorectal cancer prognosis. OMICS. 2014;18(1):54-64.

22. Sun T, Wang C, Xing J, Wu D. miR-429 modulates the expression of c-myc in human gastric carcinoma cells. Eur J Cancer. 2011;47(17): 2552-2559.

23. Li J, Du L, Yang Y, et al. MiR-429 is an independent prognostic factor in colorectal cancer and exerts its anti-apoptotic function by targeting SOX2. Cancer Lett. 2013;329(1):84-90.

24. Jay A, Parsons M. Fascin: a key regulator of cytoskeletal dynamics. Int J Cell Biol. 2010;42(10):1614-1617.

25. Adams JC. Roles of fascin in cell adhesion and motility. Curr Opin Cell Biol. 2004;16(5):590-596. 
26. Darnel AD, Behmoaram E, Vollmer RT, et al. Fascin regulates prostate cancer cell invasion and is associated with metastasis and biochemical failure in prostate cancer. Clin Cancer Res. 2009;15(4):1376-1383.

27. Hashimoto $\mathrm{Y}$, Ito $\mathrm{T}$, Inoue $\mathrm{H}$, et al. Prognostic significance of fascin overexpression in human esophageal squamous cell carcinoma. Clin Cancer Res. 2005;11(7):2597-2605.

28. Hashimoto Y, Shimada Y, Kawamura J, Yamasaki S, Imamura M. The prognostic relevance of fascin expression in human gastric carcinoma. Oncology. 2004;67(3-4):262-270.

29. Maitra A, Iacobuzio-Donahue C, Rahman A, et al. Immunohistochemical validation of a novel epithelial and a novel stromal marker of pancreatic ductal adenocarcinoma identified by global expression microarrays: sea urchin fascin homolog and heat shock protein 47. Am J Clin Pathol. 2002;118(1):52-59.

30. Pelosi G, Pastorino U, Pasini F, et al. Independent prognostic value of fascin immunoreactivity in stage I nonsmall cell lung cancer. $\mathrm{Br}$ J Cancer. 2003;88(4):537-547.
31. Rodriguez-Pinilla SM, Sarrio D, Honrado E, et al. Prognostic significance of basal-like phenotype and fascin expression in node-negative invasive breast carcinomas. Clin Cancer Res. 2006;12(5):1533-1539.

32. Machesky LM, Li A. Fascin: Invasive filopodia promoting metastasis. Commun Integr Biol. 2010;3(3):263-270.

33. Fu H, Wen JF, Hu ZL, Luo GQ, Ren HZ. Knockdown of fascin1 expression suppresses the proliferation and metastasis of gastric cancer cells. Pathology. 2009;41(7):655-660.

34. Tsai WC, Jin JS, Chang WK, et al. Association of cortactin and fascin-1 expression in gastric adenocarcinoma: correlation with clinicopathological parameters. J Histochem Cytochem. 2007;55(9):955-962.

35. Guo L, Bai H, Zou D, et al. The role of microRNA-133b and its target gene FSCN1 in gastric cancer. J Exp Clin Cancer Res. 2014; 33(1):99. 


\section{Supplementary materials}

Table SI The clinicopathologic features of the patients

\begin{tabular}{|c|c|c|c|c|c|c|c|c|}
\hline ID & Sex & Age & Tumor grades & Differentiation & $\mathbf{T}$ & $\mathbf{N}$ & $\mathbf{M}$ & Pathological types \\
\hline I & Female & 37 & IIIB & Low & 3 & $3 a$ & 0 & Adenocarcinoma \\
\hline 2 & Male & 67 & $\| \mathrm{A}$ & Low to middle & 3 & 0 & 0 & Adenocarcinoma \\
\hline 3 & Male & 58 & IIIA & Middle & 3 & 2 & 0 & Tubular adenocarcinoma \\
\hline 4 & Female & 37 & IIA & Not known & 3 & 0 & 0 & Lymphoma \\
\hline 5 & Male & 49 & IIIC & Low to middle & $4 b$ & 2 & 0 & Adenocarcinoma \\
\hline 6 & Male & 56 & IIIB & Low & 3 & $3 a$ & 0 & Adenocarcinoma \\
\hline 7 & Male & 71 & IIIA & Middle & 3 & 2 & 0 & Papillary adenocarcinoma \\
\hline 8 & Male & 57 & IV & Middle & 3 & $3 a$ & I & Adenocarcinoma \\
\hline 9 & Male & 77 & IIA & Middle & 3 & 0 & 0 & Squamous-cell carcinoma \\
\hline 10 & Male & 50 & IIIA & Low & 3 & 2 & 0 & Adenocarcinoma \\
\hline II & Male & 48 & IIIB & Low to middle & 3 & $3 a$ & 0 & Adenocarcinoma \\
\hline 12 & Female & 61 & IIIB & Low to middle & 3 & $3 a$ & 0 & Adenocarcinoma \\
\hline 13 & Male & 75 & IIA & Middle & 3 & 0 & 0 & Adenocarcinoma \\
\hline 14 & Male & 60 & IB & Low to middle & 2 & 0 & 0 & Adenocarcinoma \\
\hline 15 & Male & 66 & IIIB & Low & 3 & $3 a$ & 0 & Adenocarcinoma \\
\hline 16 & Male & 61 & IIIB & Low to middle & 3 & $3 a$ & 0 & Adenocarcinoma \\
\hline 17 & Male & 76 & IIB & Low & 3 & I & 0 & Squamous-cell carcinoma \\
\hline 18 & Male & 66 & IB & Middle & 2 & 0 & 0 & Adenocarcinoma \\
\hline 19 & Female & 49 & IIIA & Low & 3 & 2 & 0 & Adenocarcinoma \\
\hline 20 & Male & 76 & IIB & Low to middle & 3 & 1 & 0 & Adenocarcinoma \\
\hline 21 & Female & 42 & IIIB & Low & $4 a$ & 2 & 0 & Mucinous carcinoma \\
\hline 22 & Male & 69 & IB & Low & $\mathrm{la}$ & 0 & 0 & Adenocarcinoma \\
\hline 23 & Male & 62 & IIIC & Low & $4 a$ & $3 b$ & 0 & Adenocarcinoma \\
\hline 24 & Female & 78 & IIA & Middle & 3 & 0 & 0 & Adenocarcinoma \\
\hline 25 & Male & 64 & IIB & Low to middle & $4 a$ & 0 & 0 & Adenocarcinoma \\
\hline 26 & Male & 70 & IIIA & Middle & $4 a$ & I & 0 & Adenocarcinoma \\
\hline 27 & Male & 77 & IIIA & Low & $4 a$ & I & 0 & Adenocarcinoma \\
\hline 28 & Male & 49 & IIIB & Low to middle & $4 a$ & 2 & 0 & Adenocarcinoma \\
\hline 29 & Male & 34 & IIIB & Middle & $4 a$ & 2 & 0 & Adenocarcinoma \\
\hline 30 & Female & 7I & IIB & Middle & $4 a$ & 0 & 0 & Adenocarcinoma \\
\hline 31 & Male & 50 & IIIB & Middle & $4 a$ & 2 & 0 & Adenocarcinoma \\
\hline 32 & Female & 50 & IIIB & Low to middle & $4 a$ & 2 & 0 & Adenocarcinoma \\
\hline 33 & Male & 63 & IIIC & Low to middle & $4 a$ & $3 b$ & 0 & Adenocarcinoma \\
\hline 34 & Male & 66 & IIIB & Low to middle & $4 b$ & I & 0 & Adenocarcinoma \\
\hline 35 & Female & 64 & Not known & Not known & & & & Not known \\
\hline 36 & Male & 64 & $\mathrm{IIIC}$ & Low & $4 a$ & $3 b$ & 0 & Adenocarcinoma \\
\hline 37 & Female & 56 & Not known & Not known & & & & Not known \\
\hline 38 & Female & 61 & IV & Low & 3 & $3 a$ & I & Adenocarcinoma \\
\hline 39 & Male & 60 & IIIC & Middle & $4 b$ & 2 & 0 & Adenocarcinoma \\
\hline 40 & Male & 63 & IV & Low to middle & $4 b$ & I & I & Adenocarcinoma \\
\hline
\end{tabular}




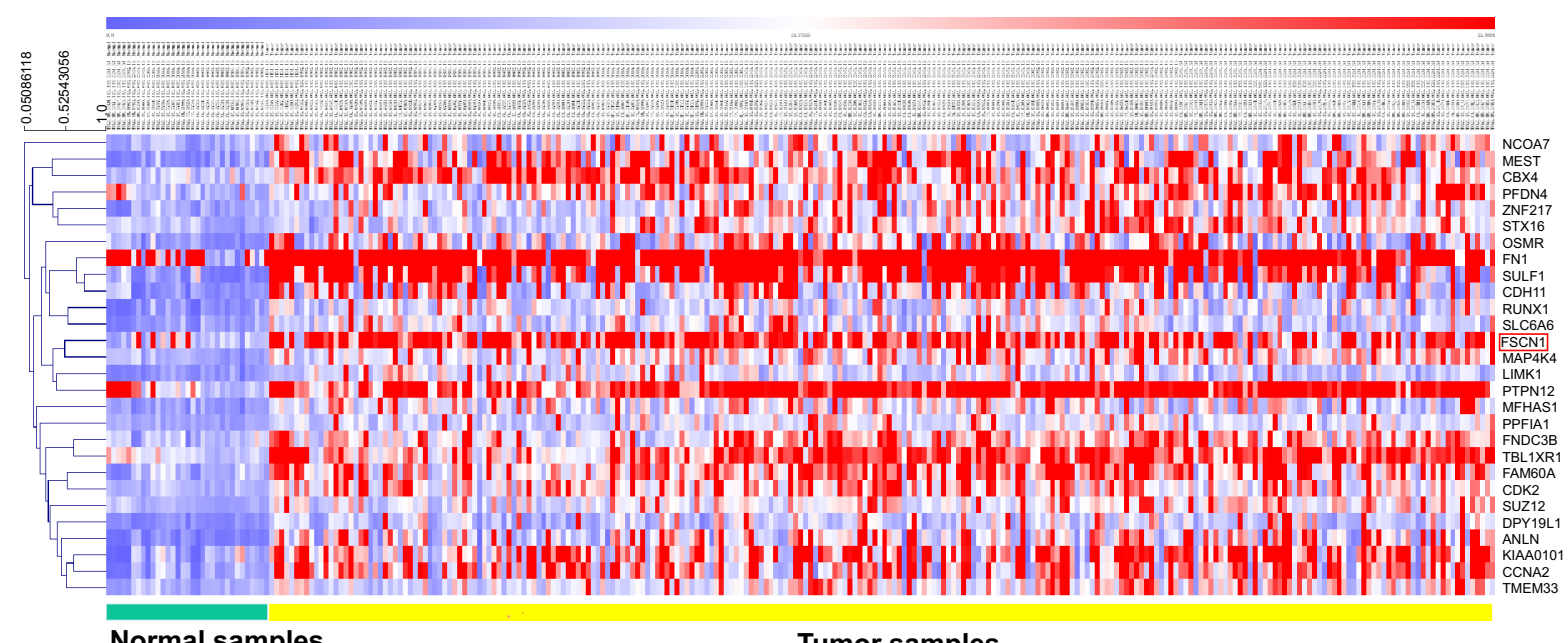

Figure SI Cluster miR-429 targets expression profile between normal tissues and gastric tumors.

Notes: Statistical analysis of the miR-429 targets between 33 normal samples and 249 gastric tumor samples predicts 168 differently expressed genes. The color scale denotes the extent of relative upregulation (red) and downregulation (blue). Green and yellow column-wise color codes for 33 normal and 249 tumor samples, respectively. Y-axis displays the gene symbol and the gene fascin-I (FSCNI) is marked within the red rectangle.

\section{Number of genes per GO term}

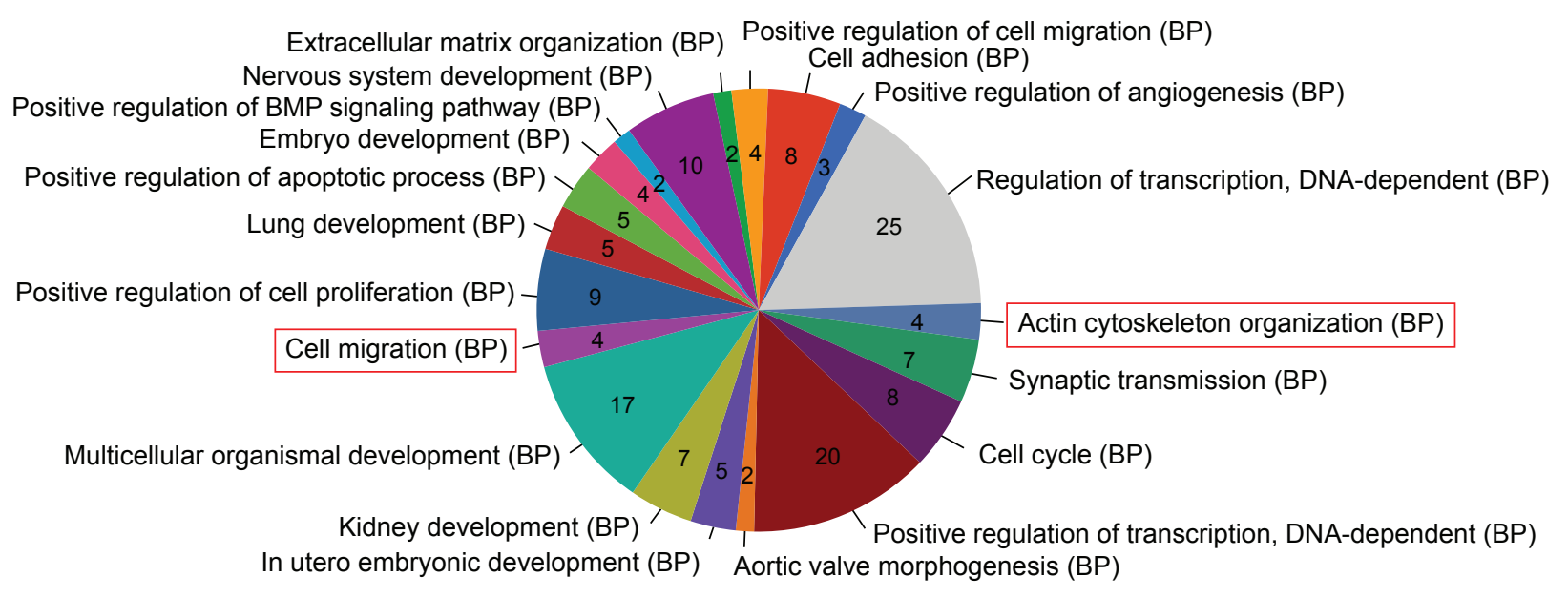

Figure S2 Gene oncology (GO) distributions after microRNA target analysis as grouped by molecular function.

Notes: The BP terms associated with fascin-I include actin cytoskeleton organization and cell migration. The genes significantly related to our research were marked by red boxes.

Abbreviations: BP, biological process; BMP, bone morphogenetic protein.

\section{Publish your work in this journal}

OncoTargets and Therapy is an international, peer-reviewed, open access journal focusing on the pathological basis of all cancers, potential targets for therapy and treatment protocols employed to improve the management of cancer patients. The journal also focuses on the impact of management programs and new therapeutic agents and protocols on patient perspectives such as quality of life, adherence and satisfaction. The manuscript management system is completely online and includes a very quick and fair peer-review system, which is all easy to use. Visit http://www.dovepress.com/testimonials.php to read real quotes from published authors. 